\title{
Empleo de Twitter en la docencia universitaria: hacia un modelo de aprendizaje basado en la co-creación.
}

Emmanuel Martínez, Eva ${ }^{\mathrm{a}}$ y Gómez Rodríguez, Pedro Manuel ${ }^{\mathrm{b}}$

${ }^{a}$ Profesora de la Escuela Universitaria de Estudios Empresariales de Bilbao, Universidad del País Vasco UPV/EHU, eva.emmanuel@ehu.eus y b Catedrático de Escuela Universitaria, Universidad del País Vasco UPV/EHU, pedro.gomez@ehu.eus

\begin{abstract}
College students are considered by some researchers "digital natives". New technologies are part of their mental structures and their way of understanding reality and information. Based on the various theories of learning, it seems appropriate to consider whether the use of social platforms like Twitter collaborates and supports the process of autonomous learning and team participation in the cocreation of knowledge, the involvement and student motivation for learning process. Following a review of previous research, the authors of this study address the use of Twitter in the university teaching field from an undirected and facilitator new perspective. The results obtained through surveys and a qualitative assessment of their tweets, support the validity of some of the statements of previous research and discard others. The analysis also suggests that Twitter has great potential in the generation of a virtual community of continuous self-learning and updated very valuable for the students. The study also proposes a series of recommendations to teachers for the implementation of Twitter to support higher education.
\end{abstract}

Keywords:

Learning, Twitter, university, co-creation, social networks, higher education

Resumen
Los estudiantes universitarios son considerados por algunos
investigadores "nativos digitales". El uso de nuevas tecnologías
forma parte de sus estructuras mentales y de su forma de comprender

(c)) EY-NC-ND 2015, Universitat Politècnica de València

Congreso In-Red (2015) 
Empleo de Twitter en la docencia universitaria: hacia un modelo de aprendizaje basado en la cocreación.

la realidad e información. Partiendo de las diversas teorías del aprendizaje parece pertinente estudiar si el uso de plataformas sociales como Twitter colaboran y apoyan el proceso de aprendizaje autónomo y en equipo, la participación en la co-creación de conocimiento, la implicación y la motivación del alumno por su proceso de aprendizaje. Tras una revisión de las investigaciones previas, los autores de este estudio abordan el uso de Twitter en el ámbito docente universitario desde una perspectiva nueva no dirigida y facilitadora. Los resultados obtenidos a través de encuestas a los alumnos y una valoración cualitativa de sus tweets, apoyan la validez de algunas de las afirmaciones de investigaciones anteriores y descartan otras. El análisis también sugiere que Twitter tiene un gran potencial en la generación de una comunidad virtual de autoaprendizaje continuo y actualizado muy valioso para el estudiante. El estudio propone además, una serie de recomendaciones a los docentes para la implementación de Twitter como apoyo a los estudios superiores.

\section{Palabras clave:}

Aprendizaje, Twitter, universidad, co-creación, redes sociales, educación superior

\section{Introducción.}

Los alumnos que llenan hoy nuestras universidades pueden considerarse, según algunos investigadores, "nativos digitales": jóvenes que han estado inmersos en la tecnología desde su nacimiento y, por tanto, dotados de unas habilidades técnicas elevadas y unas preferencias en el aprendizaje y en la búsqueda de información y conocimiento que, muchas veces, no encajan con la educación tradicional (Prensky, 2001). Pero, mientras algunos autores afirman que los nativos digitales aprenden de manera diferente, otros autores son críticos con esta idea (Bennett, Maton, y Kervin, 2008).

La Universidad deberá probar e investigar la efectividad y conveniencia académica de incorporar estos nuevos lenguajes y formas de interacción social en sus programas educativos. Deberá contrastar si co-crear con los alumnos su propio patrón de aprendizaje a través de las nuevas tecnologías provoca una mayor efectividad pedagógica e interés por parte del alumnado. Parece lógico pensar que el uso de redes sociales como Twitter y de los 
dispositivos móviles puede aportar nuevos modelos de enseñanza más participativos, dinámicos, proactivos, actualizables, transferibles y motivacionales donde el estudiante no sólo participa en el proceso de enseñanza como un mero receptor de conocimientos sino como emisor y protagonista de su propio proceso.

\subsection{Teorías del Aprendizaje y Redes Sociales.}

Hay dos tipos principales de anotaciones o inputs para el aprendizaje: la pictórica y la escrita (Al-Seghayer, 2001). Se ha visto como el uso de anotaciones de palabras para el aprendizaje (Cohen, 1981; Taylor y Taylor, 1990) y de palabras clave (Courtney, 1998; Pressley et al., 1982) requieren un procesamiento más profundo tanto para comprender el significado de las palabras como para mejorar la retención de dichas palabras objetivo. Twitter, con su especificidad de los 140 caracteres obliga a una elección continua de palabras clave que, previsiblemente, y a la vista de estudios precedentes, servirá al alumno para una mayor comprensión/recuerdo de lo aprendido. Existen investigaciones que demuestran cómo el aprendizaje visual facilitado por los soportes móviles de los alumnos, es más fácil de retener que el contenido verbal (Chen et al., 2008). El uso de estas nuevas herramientas ofrece oportunidades de mejora en la interacción entre profesores y alumnos y una mayor implicación de éstos en la asignatura (Brown, 2005).

Siguiendo las reflexiones de Hussin et al. (2012) desde el campo de la psicopedagogía podemos relacionar tres tipos de aprendizaje con la tecnología m-learning (aprendizaje a través del dispositivo móvil) las cuales, a través del presente proyecto, extendemos al uso de la red Twitter:

Aprendizaje Conductista - Se basa en el aprendizaje derivado de la asociación estímulorespuesta basado en la retroalimentación y el refuerzo. Los dispositivos móviles y el uso de Twitter pueden facilitar la retroalimentación de los alumnos al estar usando una plataforma donde los marcajes como favoritos y retweets hacen las veces de refuerzo positivo y retroalimentación constante.

Aprendizaje Constructivista - El proceso de aprendizaje es un proceso dinámico, participativo y continuo a través del cual las personas construyen nuevos esquemas partiendo de esquemas que ya poseen. Este proceso se hace relacionando los nuevos aprendizajes con aquello que ya forma parte de su estructura cognitiva (Carretero, 1997). En el actual entorno digital que rodea a los jóvenes, los dispositivos móviles y herramientas sociales como Twitter forman parte de sus estructuras mentales.

Aprendizaje Colaborativo - Los estudiantes son animados a construir su propio aprendizaje y a compartirlo con sus compañeros. Se promulga un trato de igual a igual (entre profesor y alumno y entre alumnos) en el cual escuchar diversas opiniones, soportar críticas o generarlas y participar en diálogos abiertos es la base del aprendizaje con el 
Empleo de Twitter en la docencia universitaria: hacia un modelo de aprendizaje basado en la cocreación.

objeto de conseguir un pensamiento crítico y creativo derivado de diálogos abiertos y significativos (Johnson, Johnson y Holubec, 1988). Con la utilización de redes sociales como Twitter, se favorece la creatividad, la colaboración y el compartir recursos tanto entre estudiantes como entre profesor y estudiantes.

Existen, además, otras teorías educativas a tener en cuenta en el contexto del m-learning:

Aprendizaje Conectivista - Este tipo de orientación pedagógica promulga un aprendizaje dinámico e informal en el cual el educador solo facilita e incluso desaparece en el proceso de aprendizaje (Siemens y Downes, 2008; Siemens, 2005). No hay un grupo de contenidos establecido, requiere de la autonomía del alumno y de su compromiso para crear y compartir conocimiento. Nace de las teorías del caos, de la red y la complejidad de la autoorganización de conexiones dentro de esa red (Kop, 2011). Dentro del ámbito educativo, Twitter posibilita la generación espontánea de contenidos diversos relacionados con la asignatura.

Aprendizaje desde las Teorías Cognitivas - El proceso de aprendizaje es el resultado de tres tipos de experiencias: auditiva, visual y kinestésica (Virtanen, Myllärniemi y Wallander, 2013). Algunos estudios señalan que los procesos de aprendizaje que aúnen estas tres diferentes maneras de aprender podrán obtener resultados alentadores (Dillon y Jobst, 2005; Gawboy y Greene, 2013). La posibilidad de incluir en un tweet todo tipo de contenidos (texto, imagen, audio...) genera experiencias multisensoriales.

Aprendizaje Mixto - Las redes sociales pueden colaborar en lo que viene a llamarse aprendizaje mixto: aprender a través de la escucha, por descubrimiento, haciendo y a través del debate y discusión (Treepuech, 2011). El descubrimiento de tweets, la generación de mensajes, los retweets, las respuestas a otros, etc. parecen suponer una oportunidad desde la teoría del aprendizaje mixto.

1.2. Utilidad de las redes sociales y nuevas plataformas móviles en el ámbito educativo: revisión científica.

\subsubsection{Interés, motivación e implicación del alumno.}

Muchos estudios relacionados con el aprendizaje móvil han probado cómo el uso de dispositivos móviles entre estudiantes universitarios es un medio efectivo para que éstos se interesen/motiven por el aprendizaje fomentando su interacción (Jacob e Issac, 2008; Abas, Lim y Tai-Kwan, 2009; Issham et al. 2010). Otros investigadores son críticos con el uso de los dispositivos móviles en la educación argumentando que el entusiasmo inicial es temporal (Gay et al., 2001) y que algunos alumnos no son capaces de utilizar eficazmente los dispositivos móviles durante su aprendizaje (Hsi, 2003). Por otro lado, existe una buena disposición de los estudiantes a integrar el aprendizaje móvil en la educación (Hussin et al.,

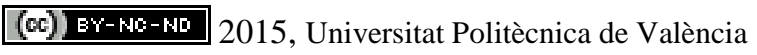


2012). Experimentar con nuevas formas de combinar el juego y las nuevas tecnologías 2.0 para mejorar o facilitar el aprendizaje es un objetivo necesario según Perifanou (2009). Las redes sociales crean sentido de pertenencia al grupo y promueven la participación (Chiu et al., 2008; Johnson y Dyer, 2008; Virtanen y Malinen, 2008; Zhang, 2010). En cuanto al uso de las redes sociales conviene señalar que muchos de los usuarios en Internet suelen utilizar la red de forma pasiva. Mientras una minoría suministra contenidos, una gran mayoría (90\%) se limita a mirar (Nielsen, 2006).

\subsubsection{Herramienta facilitadora del aprendizaje.}

Hay bastante consenso académico y científico en determinar que los dispositivos móviles (m-learning) sirven tan solo como una extensión y apoyo facilitador del aprendizaje informal, no pudiendo sustituir a las herramientas ya existentes y no pudiendo establecer una conexión directa con el aprendizaje (Chen y Chen, 2012; Ebner, 2009; Ebner et al., 2010; Gray et al., 2010; Junco, Heiberger y Loken, 2011; Liu et al., 2003; Rinaldo, Tapp y Laverie, 2011; Bueno-Delgado y Pavón-Mariño, 2012). A pesar de lo anterior, sí se ha probado que los dispositivos móviles tienen ciertas ventajas como son la independencia de una ubicación y de un tiempo concreto (Holzinger, Nischelwitzer y Meisenberger, 2005). Se afirma que el uso de redes sociales en el ámbito educativo promueve el intercambio de conocimientos y el aprendizaje informal entre los estudiantes (Forkosh-Baruch y Hershkovitz, 2012; Junco, Heiberger y Loken, 2011) y que las redes sociales académicas suelen tener una vida activa larga. Algunos autores defienden que el uso de las redes sociales en el ámbito educativo no se sostiene porque su uso es fundamentalmente informal y muchas veces no muy próximo a los objetivos concretos de aprendizaje (Selwyn, 2007). Otros autores afirman que las nuevas tecnologías de la web 2.0 son útiles para la conversación informal, el diálogo reflexivo y colaborativo en la generación de contenidos por lo que permite el acceso de los alumnos a gran cantidad de ideas de forma autónoma e independiente de los límites físicos, geográficos u organizativos (McLoughlin y Lee, 2010).

Se abre, por tanto, un campo importante para la investigación y el debate en este área así como para obtener modelos aplicados que consigan convertir estas plataformas en herramientas amigables para los propósitos educativos (Bueno-Delgado y Pavón-Mariño, 2012). Varios estudios han probado la relación entre el uso de las redes sociales en la docencia y mayores niveles de motivación, aprendizaje y clima positivo en el aula (Dillon y Jobst, 2005; Mazer et al., 2007) sobre todo cuando los alumnos creen que su uso es relevante y están debidamente motivados a usarlas en el ámbito académico (Rinaldo, Tapp y Laverie, 2011).

\subsubsection{Formas de implementación.}

Existe un debate abierto sobre el uso de las redes sociales dentro del aula o campus. Algunas voces dicen que las redes sociales dentro del aula suponen una distracción (Fox y

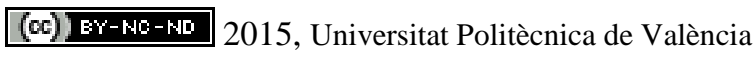

Congreso In-Red (2015) 
Empleo de Twitter en la docencia universitaria: hacia un modelo de aprendizaje basado en la cocreación.

Varadarajan, 2011). Otras creen que estas plataformas pueden ser utilizadas para facilitar una educación superior (Dhume et al., 2012). Algunas investigaciones y estudios realizados al respecto (Junco, Heiberger y Loken, 2011) exigen a los alumnos un número determinado de aportaciones y un tipo concreto de contenido mínimo. Muchos estudios reflejan cómo un número importante de alumnos no estaban familiarizados con Twitter por lo que los profesores se encontraron con una dificultad añadida para su puesta en marcha y los estudiantes con alguna dificultad para utilizarla (Agherdien, 2011; Costa et al., 2008; Rinaldo et al., 2011). En ocasiones esta dificultad ha derivado en reticencia hacia su uso por lo que algunos autores han establecido recompensas a los alumnos (Rinaldo et al., 2011). Para solventar este problema se recomienda un aprendizaje o entrenamiento previo a los alumnos para que se familiaricen con la red social que vaya a ser utilizada (Gray et al., 2010). Y como apuntan Dillon y Jobst (2005), la presencia de la figura de un facilitador/moderador es crucial en el éxito de la aplicación de las redes sociales en el aula. El papel del profesor en la utilización de Twitter y otras herramientas sociales deberá ser por tanto el de mediador, animador, orientador y evaluador crítico (Nikolidakis y Paraskevas 2012).

\subsubsection{Desventajas.}

Cuando se utilizan plataformas y tecnologías informáticas con fines educativos los profesores deben ser conscientes de que pueden darse en los estudiantes resultados positivos pero también negativos (Grosseck y Holetescu, 2008; Herrmann, Fox y Boyd, 2000; Lowe y Laffey, 2001). Entre los puntos negativos respecto a su uso están el reducido porcentaje de alumnos que finalmente son participativos frente a una mayoría de mirones (Ebner, 2009; Kop, 2011; Rinaldo, Tapp y Laverie, 2011; Ros et al., 2011); los riesgos de privacidad y mal uso (Rinaldo, Tapp. y Laverie, 2011); la sobrecarga de trabajo para el profesor y para el estudiante (Schroeder, Minocha y Schneider, 2010). Uno de los problemas apuntados por la mayoría de los autores se refiere a la dificultad del diseño de las iniciativas sociales con fines pedagógicos y de la evaluación de los contenidos y aprendizaje obtenido por los estudiantes (Gray et al., 2010).

\subsubsection{Conclusiones.}

Una revisión bibliográfica de las investigaciones existentes sobre el uso del microblogging en educación realizada por Gao, Luo y Zhang (2012) destaca como conclusión general lo siguiente: una mayoría de investigaciones se realiza dentro del ámbito del estudio de idiomas; los estudios que aplicaron el microblogging como herramienta educativa de participación inmediata en el aula destacan el aspecto negativo relativo a la falta de retroalimientación y el nerviosismo, y como positivos la posibilidad de generar respuestas rápidamente de forma dinámica y que el uso del micrologging acaba extendiéndose más allá del aula. Asimismo, en la mayoría de las investigaciones se constata el aumento de la 
participación e implicación por parte de los alumnos al verse escuchados y un aumento de las relaciones entre ellos y con el profesor (Sampaio y Sobral, 2014). Se evidencia que las relaciones con el microblogging se mantienen incluso después de finalizado el curso y que el microblogging fomenta el pensamiento reflexivo por parte de los alumnos y el aprendizaje colaborativo.

\section{Objetivos.}

\subsection{Línea 1: Valoración de la implementación de Twitter como apoyo al aprendizaje} universitario.

Objetivo principal (OP1): Conocer si la implementación de Twitter en la docencia sirve de apoyo, mejora y/o refuerzo del aprendizaje.

Objetivos secundarios: se medirá la influencia de Twitter en las siguientes dimensiones del alumno: implicación e interés en su proceso de aprendizaje; la percepción de Twitter como herramienta que aporta valor; nivel de participación y cooperación con el grupo; destrezas para el manejo de las TICs. También se medirá el grado de satisfacción tras la experiencia y el de utilidad percibida.

2.2. Línea 2: Contraste de un modelo de aplicación de Twitter en el aula basado en la co-creación como apoyo a la docencia universitaria.

Objetivo principal (OP2): Valorar la oportunidad de un modelo de aplicación de Twitter en el aula basado en la co-creación entre alumnado y profesor.

Objetivos secundarios: se llevará un registro de la cantidad de las aportaciones de los alumnos; se valorará la calidad, novedad y adecuación de las aportaciones o tweets realizados por parte de los alumnos; y se medirá la cantidad de las interacciones generadas por parte de los alumnos dentro del perfil de Twitter creado para la asignatura.

\section{Desarrollo de la innovación.}

La innovación se ha aplicado en la asignatura de Marketing Internacional del $4^{\circ}$ curso del Grado en Gestión de Negocios de la Universidad del País Vasco (UPV/EHU), que tiene carácter opcional y pertenece a la especialidad de Comercio y Marketing. En el desarrollo de la asignatura se utiliza una combinación de diferentes herramientas docentes: clases magistrales, prácticas en el aula, juego grupal de Business Game (simulación de negocios) y trabajos individuales. El uso de Twitter se presenta ante los alumnos como una actividad complementaria a las anteriormente descritas y con carácter totalmente voluntario e individual. La implantación de la innovación se ha realizado entre septiembre y diciembre de 2014 (marco temporal de la asignatura), en un grupo compuesto por 37 estudiantes.

Para facilitar el logro de los objetivos previstos, la implementación de Twitter se aborda desde una perspectiva no dirigida y facilitadora rigiéndose por los siguientes parámetros de

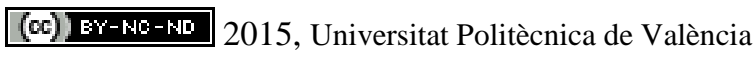

Congreso In-Red (2015) 
Empleo de Twitter en la docencia universitaria: hacia un modelo de aprendizaje basado en la cocreación.

partida: no contempla el uso de la plataforma durante las horas de docencia de la asignatura en clase sino fuera de ella, con el fin de evitar la distracción del alumnado; la participación del alumnado es voluntaria para evitar que se vean condicionados/sesgados por el puro interés calificatorio; no se exige a los alumnos un número determinado de tweets ni un tipo concreto de contenido o una cantidad mínima de participación; el profesor tan solo ejerce una labor de seguimiento, motivadora y facilitadora, aportando, en igualdad de condiciones que los alumnos, contenidos de interés de actualidad que vayan surgiendo en el mundo en relación a la materia de la asignatura, sin un esquema de contenido docente previo. Con estos parámetros podremos conocer el grado de respuesta natural de los alumnos y su nivel de implicación interno con la iniciativa así como su capacidad para co-crear libremente contenidos que se revelen de utilidad para el aprendizaje de la asignatura.

\subsection{Fases de implementación de la innovación.}

La implementación de la innovación en el aula se desarrolla en cuatro fases diferenciadas.

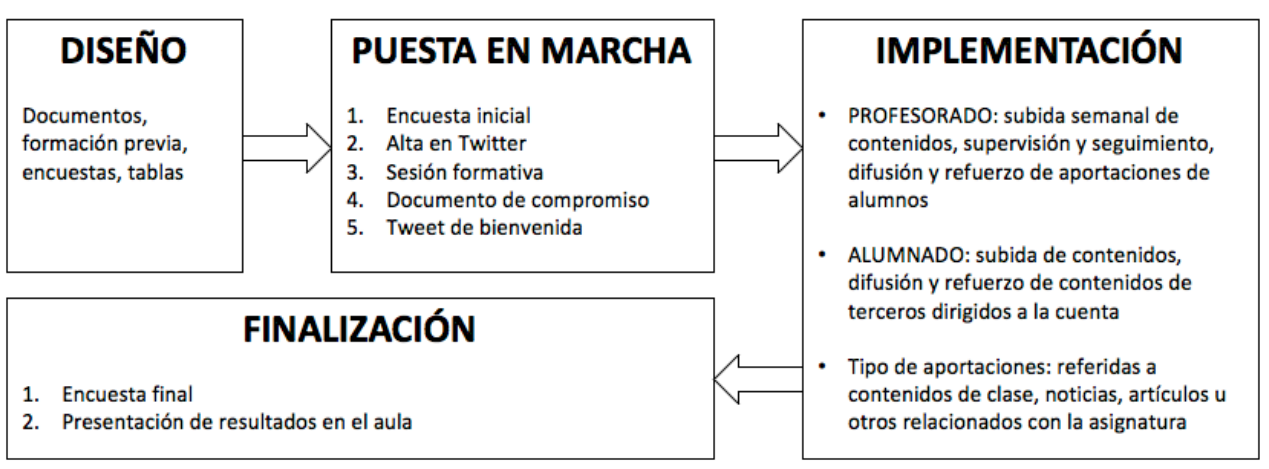

Fig. 1 Fases de la implementación de la innovación.

\section{Resultados.}

\subsection{Metodología.}

Para la consecución de los objetivos propuestos, hemos llevado a cabo un estudio exploratorio basado en un análisis previo de la bibliografía existente y en dos estudios de campo organizados en dos líneas de trabajo complementarias:

4.1.1. Línea de trabajo 1: Valoración de la implementación de Twitter como apoyo al aprendizaje universitario.

Medición mediante encuesta, en dos momentos diferentes (antes y después de la implementación de la acción) de una serie de variables relacionadas con los objetivos de la investigación, utilizando la escala de Likert. Se mide el grado de acuerdo (1 total desacuerdo, 5 total acuerdo) respecto a la influencia del empleo de Twitter en diferentes 
dimensiones relacionadas con los objetivos. Asimismo, en el cuestionario final se incluyeron tres preguntas para determinar el grado de satisfacción e interés de los alumnos y su percepción acerca de la utilidad de esta acción de innovación como apoyo al aprendizaje de la asignatura.

4.1.2. Línea de trabajo 2: Contraste de un modelo de aplicación de Twitter en el aula basado en la co-creación como apoyo a la docencia universitaria.

Valoración cuantitativa y cualitativa, mediante el seguimiento y evaluación de los contenidos libremente generados por los alumnos, empleando la siguiente tabla de registro:

Tabla 1. Tabla de registro de la Línea de trabajo 2.

\begin{tabular}{|c|c|c|c|c|c|c|c|c|c|c|c|}
\hline \multirow{3}{*}{$\begin{array}{c}\begin{array}{c}\text { Criterio 1 } \\
\text { Cuantitativo }\end{array} \\
\mathrm{N}^{\circ} \text { tweets }\end{array}$} & \multicolumn{9}{|c|}{ Criterio 2 Cualitativo } & \multirow{2}{*}{\multicolumn{2}{|c|}{$\begin{array}{c}\text { Criterio } 3 \\
\text { Interactivo }\end{array}$}} \\
\hline & \multicolumn{3}{|c|}{ Calidad } & \multicolumn{3}{|c|}{ Novedad } & \multicolumn{3}{|c|}{ Aportación } & & \\
\hline & Alta & Media & Baja & Alta & Media & Baja & Alta & Media & Baja & $\begin{array}{c}\text { Interac. } \\
\text { propia }\end{array}$ & $\begin{array}{c}\text { Interac. } \\
\text { generada }\end{array}$ \\
\hline $\begin{array}{l}\mathrm{N}^{\circ} \text { de tweets } \\
\text { publicados } \\
\text { por el } \\
\text { alumno }\end{array}$ & \multicolumn{3}{|c|}{$\begin{array}{l}\text { Acierto, seriedad, } \\
\text { profesionalidad, } \\
\text { rigurosidad, del contenido, } \\
\text { criterio y buen ajuste al } \\
\text { campo de conocimiento } \\
\text { del marketing y la empresa }\end{array}$} & \multicolumn{3}{|c|}{$\begin{array}{l}\text { Grado de novedad de los } \\
\text { contenidos respecto a lo } \\
\text { impartido en clase }\end{array}$} & \multicolumn{3}{|c|}{$\begin{array}{l}\text { Capacidad para aportar } \\
\text { valor y un mejor } \\
\text { conocimiento o } \\
\text { ejemplarización de lo } \\
\text { expuesto en clase }\end{array}$} & \multicolumn{2}{|c|}{$\begin{array}{l}\text { Número de } \\
\text { interacciones que } \\
\text { genera el alumno }\end{array}$} \\
\hline
\end{tabular}

Fuente: elaboración propia.

Considerando estas dos líneas de trabajo se obtienen tres mediciones: antes del empleo de Twitter (encuesta alumnos); durante el empleo de Twitter (cantidad, calidad, novedad, oportunidad e interacción de los contenidos generados por los alumnos); tras el empleo de Twitter (encuesta alumnos).

\subsection{Resultados obtenidos.}

Los resultados se han obtenido utilizando el software SPSS y Microsoft Excel. La actividad ha tenido en líneas generales, los siguientes índices de participación:

Tabla 2. Índices de participación del alumnado.

\begin{tabular}{|c|c|c|c|}
\hline Alumnos que participan voluntariamente & $85 \%$ & $\begin{array}{l}\text { Los alumnos activos en la } \\
\text { participan: }\end{array}$ & cuenta \\
\hline \multirow{2}{*}{$\begin{array}{l}\text { Entre los alumnos que leen los tweets: } \\
\text { Leen la mayoría de los tweets }\end{array}$} & \multirow[b]{2}{*}{$30 \%$} & \multirow{2}{*}{$\begin{array}{r}\text { Aportando tweets } \\
\text { Marcando favoritos y retwitteando }\end{array}$} & $59 \%$ \\
\hline & & & $23 \%$ \\
\hline \multirow{2}{*}{$\begin{array}{r}\text { Leen aproximadamente la mitad de los tweets } \\
\text { Leen muy pocos tweets }\end{array}$} & \multirow{2}{*}{$\begin{array}{l}35 \% \\
15 \%\end{array}$} & Leyendo tweets & $18 \%$ \\
\hline & & $\begin{array}{r}\text { Alumnos pasivos "mirones" en la cuenta } \\
\text { Alumnos activos en la cuenta }\end{array}$ & $\begin{array}{l}21 \% \\
79 \%\end{array}$ \\
\hline
\end{tabular}

4.2.1. Línea de trabajo 1: Valoración de la implementación de Twitter como apoyo al aprendizaje universitario.

Los resultados obtenidos se resumen en la tabla 3, en la que se muestra la diferencia de medias observada entre las dos mediciones, y el valor de la significación bilateral, que

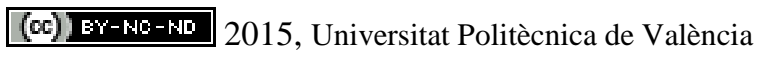

Congreso In-Red (2015) 
Empleo de Twitter en la docencia universitaria: hacia un modelo de aprendizaje basado en la cocreación.

compararemos con el valor 0,05 para rechazar o no la hipótesis nula de igualdad de medias, tras aplicar el contraste t-Student para medias independientes.

Respecto a las dimensiones analizadas en la Línea 1, a pesar de que se aprecia una mejora en las valoraciones medias de todas las variables analizadas, sólo en algunos casos la diferencia observada es significativa. Se verifica la aportación de Twitter en el aprendizaje de los alumnos y en la destreza en el manejo de las TICs. Por otra parte, se ha obtenido un elevado índice de satisfacción en su uso y en la percepción del alumnado como herramienta de apoyo en su proceso de aprendizaje.

Tabla 3. Resultados de la comparación entre las dos encuestas.

\begin{tabular}{|c|c|c|c|c|}
\hline Variable del cuestionario & $\begin{array}{l}\text { Dimensiones a las que se } \\
\text { refiere }\end{array}$ & $\begin{array}{l}\text { Media } \\
\text { inicial }\end{array}$ & Media final & $\begin{array}{l}\text { Sig. } \\
\text { bilateral* }\end{array}$ \\
\hline $\begin{array}{l}\text { 10. Cuál ha sido tu nivel de participación en la realización } \\
\text { de propuestas de conocimiento relacionados con las clases. }\end{array}$ & \multirow{2}{*}{$\begin{array}{l}\text { Implicación e interés del } \\
\text { alumno }\end{array}$} & 3,189 & 3,385 & 0,361 \\
\hline $\begin{array}{l}\text { 12. Estoy dispuesto a asumir esfuerzo en mi proceso de } \\
\text { aprendizaje. }\end{array}$ & & 4,405 & 4,615 & 0,293 \\
\hline $\begin{array}{l}\text { 24. Soy capaz de buscar y encontrar fácilmente información } \\
\text { útil para la gestión del marketing internacional de una } \\
\text { empresa. }\end{array}$ & $\begin{array}{l}\text { Aportación al } \\
\text { aprendizaje }\end{array}$ & 2,946 & 3,615 & 0,049 \\
\hline $\begin{array}{l}\text { 9. Cuál ha sido tu grado de colaboración habitual con los } \\
\text { compañeros en la creación de contenidos relacionados con } \\
\text { las asignaturas. }\end{array}$ & \multirow{4}{*}{$\begin{array}{l}\text { Participación y } \\
\text { cooperación }\end{array}$} & 3,56 & 3,769 & 0,443 \\
\hline $\begin{array}{l}\text { 11. Cuál ha sido tu nivel de cooperación con los } \\
\text { compañeros de clase en el debate y en la participación en } \\
\text { discusiones de grupo sobre contenidos relacionados con las } \\
\text { asignaturas. }\end{array}$ & & 3,324 & 4,154 & 0,002 \\
\hline $\begin{array}{l}\text { 27. Me gusta aportar ideas y propuestas a mis } \\
\text { compañeros/as de asignatura. }\end{array}$ & & 3,62 & 3,923 & 0,255 \\
\hline $\begin{array}{l}\text { 28. Me siento cómodo expresando mis opiniones e ideas al } \\
\text { resto de mis compañeros. }\end{array}$ & & 3,649 & 4,077 & 0,107 \\
\hline 20. Manejo con soltura algunas plataformas sociales. & \multirow{3}{*}{$\begin{array}{c}\text { Destreza en el manejo de } \\
\text { las TICs }\end{array}$} & 3,649 & 4,308 & 0,028 \\
\hline 21. Las redes sociales son valiosas para el estudio. & & 3,7 & 4,308 & 0,079 \\
\hline $\begin{array}{l}\text { 22. Las redes sociales son valiosas para la gestión } \\
\text { empresarial. }\end{array}$ & & 4,0 & 4,692 & 0,021 \\
\hline ¿Cómo valorarías la experiencia? & \multirow{2}{*}{ Satisfacción del alumno } & \multicolumn{3}{|c|}{$90 \%$ Buena y Muy buena } \\
\hline ¿Te ha parecido interesante? & & \multicolumn{3}{|c|}{$85 \%$ Interesante y Muy interesante } \\
\hline $\begin{array}{l}\text { ¿Crees que te ha ayudado a una mejor comprensión y } \\
\text { adquisición de conocimientos relacionados con el campo } \\
\text { del marketing? }\end{array}$ & $\begin{array}{l}\text { Percepción como } \\
\text { herramienta de apoyo }\end{array}$ & \multicolumn{3}{|c|}{$85 \%$ Bastante y Mucho } \\
\hline
\end{tabular}

* Se asume igualdad de varianzas, tras la aplicación de la prueba de Levene.

4.2.2. Línea de trabajo 2: Contraste de un modelo de aplicación de Twitter en el aula basado en la co-creación como apoyo a la docencia universitaria.

El número de tweets inapropiados o no relacionados con la asignatura y el campo de conocimiento del marketing ha sido nulo lo cual muestra la seriedad con la que los estudiantes han abordado el proyecto. La publicación de los tweets de los alumnos se ha producido fuera del horario de la asignatura sin preferencia por día concreto u hora. Los alumnos, por tanto, se mantienen activos en la cuenta de Twitter de la asignatura en todo momento, fuera incluso del horario académico. 
Se aprecia en la tabla 4 un elevado volumen de aportaciones, pero un gran desequilibrio en el $n^{\circ}$ de aportaciones por alumno, ya que un $26 \%$ de los alumnos aporta el $80 \%$ de los tweets. Vista la calidad, novedad y aportación de los alumnos, se constata que toda su cocreación se ha dado exclusivamente en el ámbito de la asignatura en particular y también en el campo de conocimiento general del marketing. Por otra parte, el número de interacciones generadas no ha sido elevado, confirmándose que los alumnos son más proclives a generar/aportar contenidos que a utilizar/reforzar contenidos vertidos por sus compañeros.

Tabla 4. Resultados de la revisión de las aportaciones de los alumnos.

\begin{tabular}{|c|c|c|c|c|c|c|}
\hline \multicolumn{2}{|c|}{$\begin{array}{l}\text { 1. Cuantitativo: } \\
\text { Cantidad de aportaciones }\end{array}$} & \multicolumn{4}{|c|}{$\begin{array}{l}\text { 2. Cualitativo: } \\
\text { Calidad, novedad y aportación }\end{array}$} & 3. Interactivo \\
\hline $\mathrm{N}^{0}$ tweets & 348 & & Alta & Media & Baja & \multirow{5}{*}{$\begin{array}{c}93 \text { interacciones } \\
\text { (26\% de los } \\
\text { tweets } \\
\text { publicados) }\end{array}$} \\
\hline Generados por alumnos & $66,37 \%$ & Calidad & $61 \%$ & $32 \%$ & $7 \%$ & \\
\hline $\begin{array}{l}\text { Media de tweets diarios } \\
\text { alumnos }\end{array}$ & 3,3 & Novedad & $69 \%$ & $21 \%$ & $1 \%$ & \\
\hline $\begin{array}{l}\text { Media de tweets por alumno } \\
\text { Desv. Típica } \\
\text { Moda }\end{array}$ & $\begin{array}{c}10 \\
14,62 \\
1\end{array}$ & $\begin{array}{l}\text { Aportación a la } \\
\text { asignatura }\end{array}$ & $54 \%$ & $34 \%$ & $12 \%$ & \\
\hline $\mathrm{N}^{\mathrm{o}}$ de tweets por alumno & Entre 1 y 43 & $\begin{array}{l}\text { Aportación al } \\
\text { campo de la } \\
\text { asignatura }\end{array}$ & $93 \%$ & $6 \%$ & $1 \%$ & \\
\hline
\end{tabular}

\section{Conclusiones.}

A la vista de los resultados obtenidos en el presente estudio, se confirman varias de las investigaciones precedentes a este estudio realizadas en el campo del aprendizaje a través de Twitter en la educación superior. Se constata que el uso de Twitter es percibido como una herramienta de apoyo al aprendizaje teórico y práctico obtenido de los contenidos de la asignatura y su campo de conocimiento. También queda reforzada su percepción de mejora obtenida en el manejo de las redes sociales. Una vez finalizada, la percepción de los alumnos con la experiencia de aplicación de Twitter en el desarrollo de la asignatura ha sido significativamente positiva e interesante. No se ha podido comprobar en el presente estudio que el uso de Twitter haya aumentado el grado de implicación personal, interés, curiosidad y motivación por la asignatura en particular.

La oportunidad que supone la aplicación de Twitter para la co-creación de contenidos entre alumnado y profesor queda demostrada en este trabajo por los siguientes hechos: se ha respetado en todo momento el campo de conocimiento de la asignatura; las numerosas aportaciones realizadas por los estudiantes poseen una elevada calidad; la experiencia de aplicación ha trascendido el marco temporal y espacial de la asignatura, debido al gran interés y a la motivación que ha generado el empleo de Twitter como herramienta docente. Se confirman por tanto teorías precedentes al respecto: los estudiantes no interactuaron mucho con los tweets de otros; el desequilibrio en las aportaciones confirma la teoría de que

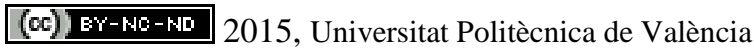

Congreso In-Red (2015) 
Empleo de Twitter en la docencia universitaria: hacia un modelo de aprendizaje basado en la cocreación.

una gran mayoría de usuarios son pasivos; el mantenimiento de un grupo de alumnos vinculados e interactuando con ella después del estudio, fuera del horario académico y una vez acabado el curso. Finalmente, no se confirman investigaciones precedentes acerca del riesgo de la arbitrariedad de los tweets subidos por los alumnos aportando contenidos personales, no relevantes, ofensivos, inapropiados o no relacionados con la materia.

Una aportación nueva de presente estudio es la aplicación de Twitter de una forma voluntaria, facilitadora y no dirigida. Lo que aporta esta investigación es el conocimiento de la disposición natural de los alumnos a participar del aprendizaje mediante el uso de Twitter. Podemos afirmar, al abrigo de los resultados en el presente estudio, que los estudiantes, por medio del uso de Twitter, co-crean su propio proceso de aprendizaje de forma colaborativa en un ambiente de aportación conectivista donde el profesor casi desaparece, o tan solo facilita, sin influir en los temas y contenidos compartidos por los alumnos. Queda contrastada en la línea de investigación 2 del presente estudio que aplicar Twitter en la docencia constituye por sí misma una comunidad de contenidos valiosos en el campo de la asignatura creados y dirigidos por los propios alumnos convirtiéndose éstos en protagonistas de su propio proceso de aprendizaje.

Tras la experiencia podemos afirmar que se trata de una herramienta complementaria atractiva, informal, actual y novedosa que puede añadir cierto valor, variedad y multicanalidad al proceso de aprendizaje.

A la vista de los resultados de este estudio, a la hora de aplicar Twitter en la docencia universitaria recomendamos realizar un diagnóstico previo sobre la disposición del alumnado, plantear alguna sesión de formación inicial, diseñar un documento de compromiso con las normas de utilización, llevar un registro privado con los datos de los participantes, no utilizar Twitter en el aula y mantener una actitud proactiva que incentive el uso de la cuenta de la asignatura. Los resultados exploratorios obtenidos pretenden servir de base para la aproximación e incentivación a la creación de un modelo aplicado y operativo de gestión del aprendizaje basado en la co-creación por medio de la implementación de Twitter como herramienta de apoyo al aprendizaje en la universidad.

En cuanto a las limitaciones es este trabajo y nuevas investigaciones, señalamos que el número de estudiantes participantes en este estudio es pequeño, y lo componen estudiantes de último curso de Grado. Los resultados obtenidos deben considerarse, por tanto con precaución. Sería interesante conocer si los resultados son comparables en un grupo más numeroso de estudiantes y en alumnos de los primeros cursos universitarios. Otra limitación radica en que las encuestas pre y post participación en el proyecto Twitter fueron anónimas. Esto no nos permitió comprobar si existe una relación positiva entre el uso de Twitter y el rendimiento académico en la asignatura y verificar la solidez de estudios precedentes (Junco, Heiberger y Loken, 2011). Estudios precedentes indican la falta de 
concordancia entre grupos de distintos países y los resultados obtenidos con relación a la aceptación tecnológica del uso de Twitter (Saeed y Sinnappan, 2011). Un estudio comparativo en grupos de estudiantes universitarios en centros de diferentes países sería interesante al objeto de poder demostrar la validez de los resultados obtenidos por el uso de Twitter en diferentes culturas. Nuevas investigaciones sobre los beneficios del uso de Twitter en el aprendizaje podrían ser recomendables en estudios a distancia o no presenciales, intercambios internacionales, etc.

\section{Referencias}

ABAS, Z. W., LIM, T. S. K. y TAI-KWAN, W. (2009). "Mobile learning initiative through SMS: A formative evaluation”. ASEAN Journal of Open and Distance Learning, 1(1), 49-58.

AL-SEGHAYER, K. (2001). "The effect of multimedia annotation modes on L2 vocabulary acquisition: A comparative study”. Language Learning y Technology, 5(1), 202-232.

AGHERDIEN, N. (2011). "Twitter and Edulink: balancing passive consumption with knowledge creation. Balcaen (Ed.)”, Proceedings of the 6th international conference on e-learning (pp 489 - 492). Reading: Academic Conferences, Ltd.

BENNETT, S., MATON, K., y KERVIN, L. (2008). “The 'digital natives' debate: A critical review of the evidence”. British journal of educational technology, 39(5), 775-786.

BROWN, T.H. (2005). “Towards a model for m-learning in Africa”. International Journal on E-learning, 4(3), 299-315.

BUENO-DELGADO, M. V. y PAVON-MARINO, P. (2012). "The use of social networks as support tools to improve teaching-learning process in universities”. Edulearn12: 4th International Conference on Education and New Learning Technologies, 35-44.

CARRETERO, M. (1997). “¿Qué es el constructivismo? Constructivismo y educación”. Desarrollo cognitivo y aprendizaje. Progreso. México. pp. 39-71

CHEN, L. y CHEN T-L. (2012). "Use of Twitter for formative evaluation: reflections on trainer and trainees'experiences”, British Journal of Educational Technology, 43, pp. 49-52, 2012. doi:10.1111/j.14678535.2011.01251.X

CHEN, N. S., HSIEH, S. W. y KINSHUK, A. (2008). "Effects of short-term memory and content representation type on mobile language learning”. Language learning \& technology, 12(3), 93-113.

CHIU, P.-Y., CHEUNG, CMK. y LEE, MKO. (2008). Online social networks: Why do “we” use Facebook?. Communications in Computer and Information Science, 19 (2008), pp. 67-74

COHEN, A. D., y APHEK, E. (1981). "Basifying second language learning”. Studies in Second Language Acquisition, 3(2), 221-236.

COSTA, C. et al. (2008). "Microblogging In technology enhanced learning: a use-case inspection of PPE summer school 2008”. Paper presented at the Workshop at the European Conference on Technology Enhanced Learning (ECTEL), Maastricht, the Netherlands.

http://www.know-center.tugraz.at/download_extern/papers/2008_ccosta_microblogging.pdf

COURTNEY, S. M. et al. (1998). "An area specialized for spatial working memory in human frontal cortex”. Science, 279(5355), 1347-1351.

DHUME, S. M., et al. (2012). "Adoption of social media by business education students: Application of technology acceptance model (TAM)” doi:10.1109/ICTEE.2012.6208609

DILLON, A. y JOBST, J. (2005). "Multimedia learning with hypermedia”. MAYER, R. (Ed.), The Cambridge Handbook of Multimedia Learning, Cambridge University Press, Cambridge, MA, pp. 569-588.

EBNER, M. et al. (2010). "Microblogs in higher education - A chance to facilitate informal and process-oriented learning?”. Computers \& Education, 55(1), 92-100. doi:http://dx.doi.org/10.1016/j.compedu.2009.12.006

EBNER, M. (2009). "Introducing live microblogging: how single presentations can be enhanced by the mass". Journal of Research in Innovative Teaching, 2, 1, 91-100.

\section{(c) EY-NC-ND 2015, Universitat Politècnica de València}


Empleo de Twitter en la docencia universitaria: hacia un modelo de aprendizaje basado en la cocreación.

http://www.nu.edu/assets/resources/pageResources/7638_JournalofResearch09.pdf\#page=96

FORKOSH-BARUCH, A., y HERSHKOVITZ, A. (2012). "A case study of israeli higher-education institutes sharing scholarly information with the community via social networks". The Internet and Higher Education, 15(1), 58-68.

FOX, B. I., y VARADARAJAN, R. (2011). "Use of twitter to encourage interaction in a multi-campus pharmacy management course”. American Journal of Pharmaceutical Education, 75(5), 88.

GAO, F., LUO, T., y ZHANG, K. (2012). "Tweeting for learning: A critical analysis of research on microblogging in education published in 2008-2011”. British Journal of Educational Technology, 43(5), $783-801$. doi:10.1111/j.1467-8535.2012.01357.x

GAY, G. et al. (2001). “The effects of wireless computing in collaborative learning environments”. International Journal of Human-Computer Interaction, 13(2), 257-276.

GAWBOY, A. y GREENE, A. (2013). "Teaching students with different learning styles and levels of preparation”. http://www.yale.edu/graduateschool/teaching/learningstyles.html (consultado en abril de 2015).

GRAY, K. et al. (2010). "Students as Web 2.0 authors: Implications for assessment design and conduct". Australasian Journal of Educational Technology. Vol 26, No 1 (2010)

http://ascilite.org.au/ajet/submission/index.php/AJET/article/view/1105

GROSSECK, G., y HOLETESCU, C., (2008). “Can we use Twitter for educational activities?”. 4th International Scientific Conference: eLearning and software for education, 2008.

HERRMANN, A., FOX, R. y BOYD, A. (2000). “Unintended effects in using learning technologies”. Language Learning y Technology, 12 (3) (2008), pp. 3-9

HOLZINGER, A., NISCHELWITZER, A. y MEISENBERGER, M. (2005). "Lifelong-learning support by mlearning: Example scenarios”. ACM eLearn Magazine, 5, 12.

HSI, S. (2003). “A study of user experiences mediated by nomadic web content in a museum”. Journal of Computer Assisted Learning, 19(3), 308-319.

HUSSIN, S. et al. (2012). "Mobile learning readiness among Malaysian students at higher learning institutes”. Asian Social Science, 8(12), p276.

ISSHAM, I. et al. (2010, April). “Acceptance on Mobile Learning via SMS: A Rasch Model Analysis”. International Journal of Interactive Mobile Technology, 4(2).

JACOB, S. M., y ISSAC, B. (2008). “The Mobile Devices and its Mobile Learning Usage Analysis”. International Journal of Interactive Mobile Technology, 2(1), 10-18.

JOHNSON, J. y DYER, J. (2008). “The development of formal and informal learning online through online communities of practice and social networking”. Proceedings of the 7th European Conference on Multimedia and ICT in Education, Agia Napa, Cyprus (2008)

JOHNSON, D. W., JOHNSON, R. T., y HOLUBEC, E. J. (1988). Cooperation in the classroom. Interaction Book Co.

JUNCO, R., HEIBERGER, G., y LOKEN, E. (2011). "The effect of twitter on college student engagement and grades”. Journal of Computer Assisted Learning, 27(2), 119-132.

KOP, R. ( 2011 ). “The challenges to connectivist learning on open online networks: learning experiences during a massive open online course”. International Review of Research in Open and Distance Learning, 12 , 3 , 19 - 38 .

LIU, T. C. et al. (2003). "Wireless y mobile technologies to enhance teaching y learning”. Journal of Computer Assisted Learning, 19(3), 371-382.

LOWE, B. y LAFFEY, D. (2011). "Is Twitter for the birds? Using Twitter to enhance student learning in a marketing course”. Journal of Marketing Education, vol. 33, pp. 183-192, 2011.

http://jmd.sagepub.com/content/early/2011/02/28/0273475311410851.full.pdf+html

MAYER, R. E. y SIMS, K. (1994). "For whom is a picture worth a thousand words? Extensions of a dualcoding theory of multimedia learning”. Journal of Educational Psychology, 86(3), 389-401.

MAZER, J. P., MURPHY, R. E., y SIMONDS, C. J. (2007). “I'll see you on “Facebook”: The effects of computermediated teacher self-disclosure on student motivation, affective learning, and classroom climate”. Communication Education, 56(1), 1-17. doi:10.1080/03634520601009710 
MCLOUGHLIN, C., y LEE, M. J. W. (2010). "Personalised and self regulated learning in the web 2.0 era: International exemplars of innovative pedagogy using social software”. Australasian Journal of Educational Technology, 26(1), 28-43.

MENKHOFF, T., MING, G. Y. Q., y BENGTSSON, M. L. (2012). In ARNEDILLO SANCHEZ I. I.,P. (Ed.), "Making (more) pedagogical sense of twitter with a newly developed tracking device and prioritised discussion points: The story of a collaborative twitter improvement project”. IADIS International Conference Mobile Learning, Berlin.

NIKOLIDAKIS, S., y PARASKEVAS, T. (2012). "Digital platforms, social networks, internet: The challenge in greek education”. Inted2012: International Technology, Education and Development Conference, 6259-6265.

NIELSEN, J. (2006). Participation inequality: Encouraging more users to contribute.

http://www.useit.com/alertbox/participation_inequality.html (2006) [extraido en marzo de 2015].

PERIFANOU, M. A. (2009). "Language micro-gaming: Fun and informal microblogging activities for language learning”. En Best Practices for the Knowledge Society. (pp. 1-14). Springer Berlin Heidelberg.

PRENSKY, M. (2001). “Digital natives, digital immigrants part 1”. On the horizon, 9(5), 1-6.

PRESSLEY, M., LEVIN, J. R., y MILLER, G. E. (1982). “The keyword method compared to alternative vocabulary learning strategies”. Contemporary Educational Psychology, 7, 50-60.

RINALDO, SB , TAPP, S. y LAVERIE, DA (2011). "Learning by tweeting: using Twitter as a pedagogical tool”. Journal of Marketing Education, 33 , 2 , 193 - 203 . DOI: 10.1177 / 0273475311410852 .

http://jmd.sagepub.com/content/33/2/193.full.pdf+html

ROSS, C. et al. (2011). "Enabled backchannel: conference Twitter use by digital humanists”. Journal of Documentation, 67 , 2 , 214 - 237 . DOI: 10.1108 / 00220411111109449

http://www.emeraldinsight.com/doi/full/10.1108/00220411111109449

SAEED, N., y SINNAPPAN, S. (2011). In KOMMERS, P. JI-PING ZHANG Issa, T. ISALAS,P. (Ed.). iTWEET UTWEET: The role of intrinsic and extrinsic motivation in the adoption of twitter in an E-commerce unit. Proceedings of the 2011 International Association for Development of the Information Society (IADIS) International Conference on Internet Technologies and Society (ITS 2011), Shanghai, China.

SAMPAIO, J. M. S., y SOBRAL, S. R. (2014). “Tweeting in an educational community”. In Information Systems and Technologies (CISTI), 2014 9th Iberian Conference on (pp. 1-6). IEEE.

http://ieeexplore.ieee.org/stamp/stamp.jsp?tp=yarnumber=6877063

SCHROEDER, A., MINOCHA, S., y SCHNEIDER, C. (2010). "The strengths, weaknesses, opportunities and threats of using social software in higher and further education teaching and learning". Journal of Computer Assisted Learning, 26(3), 159-174.

SELWYN, N. (2007). "Web 2.0 applications as alternative environments for informal learning-a critical review". In Paper for CERI-KERIS International Expert Meeting on ICT and Educational Performance (pp. 16-17).

SIEMENS, G., y DOWNES, S. (2008). Connectivism y connective knowledge. Universidad de Manitoba. . Obtenido en marzo 2015 de: http://tc.umanitoba.ca/connectivism/

SIEMENS, G. (2005). “Connectivism: A learning theory for the digital age”. International journal of instructional technology and distance learning, 2(1), 3-10.

TAYLOR, I., y TAYLOR, M. (1990). Psycholinguistics: Learning and using language. Englewood Cliffs, NJ: Prentice Hall.

TREEPUECH, W. (2011). In SHAOZI LI YING DAI (Ed.). The application of using social networking sites with available online tools for teaching and learning management. doi:10.1109/ITiME.2011.6130844

VIRTANEN, P., MYLLARNIEMI, J., y WALLANDER, H. (2013). "Diversifying higher education: Facilitating different ways of learning”. Campus-Wide Information Systems, 30(3), 201-11. doi:10.1108/10650741311330384

VIRTANEN, T., y MALINEN, S. (2008, October). "Supporting the sense of locality with online communities". In Proceedings of the 12th international conference on Entertainment and media in the ubiquitous era (pp. 145149). ACM.

ZHANG, Z. (2010). "Feeling the sense of community in social networking usage", IEEE Transactions on Engineering Management, 57(2), 225-239.

(c)) EY-NC-ND 2015, Universitat Politècnica de València

Congreso In-Red (2015) 